\title{
Observations of nuclei in cavitating flows
}

\author{
JOSEPH KATZ and ALLAN ACOSTA
}

California Institute of Technology, Pasadena, CA 91125, USA

\section{Introduction}

The present report focuses on the source of the large difference between the theoretical strength of pure liquid [12] and the actual tension that is required to initiate cavitation in technical fluids such as test facilities and natural waters. This discrepancy is commonly explained by the existence of nuclei, either solid particles or vapor and gas bubbles that permit phase transition to take place near equilibrium. The existence of these nuclei, their source and lifetimes have occupied much space in the technical literature for decades $[1,2,3,8]$. Yet, direct observations of tests in applications to naval hydrodynamics and hydraulic machinery flows has not provided much information about these nuclei. Their existence, however, and their effect on cavitation is in no doubt as is demonstrated by the series of photographs of a propeller that were taken in the 'Vacu-Tank' of NSMB [9] shown in Figure 1. There, addition of 'nucleating' sources to the water by electrolysis clearly increases the number of visible cavitating bubbles on the blade surfaces.

The importance of these nuclei has led to the development of several detection and observation techniques $[4,6,10,11]$. These include the microscopic observations of water samples, the 'coulter counter', 'single particle light scattering', the 'acoustic' techniques for detection of gas bubbles, holograms with microscopic observation of the reconstructed image, and finally the venturi 'liquid quality' meter [11].

Some results of the nuclei number density distribution function $N(R)$ that were accumulated from various sources [6] in different experimental facilities and some open sea measurements are presented in Figure 2. The results include holographic observations in the Caltech HSWT which are found to be mostly solid particles and the Caltech LTWT which are found to be mostly bubbles. This difference in the type of nuclei may explain why is it easier to cavitate the same body in the LTWT (provided that the flow is non-separating) while the liquid in the HSWT can support a certain tension [6]. Note the several orders of magnitude difference in the populations shown and the correspondence between the oceanic acoustic measurements of Medwin [10] that display the population of bubbles and the holographic observations of plankton $[5,13]$. It is interesting that some of these facilities are well above the 'natural' levels and it may be inferred that the Vacu-Tank may be well below. Thus, significant 'scaling' errors may occur while applying laboratory results to field cavitation phenomena $[1,2,3]$. 


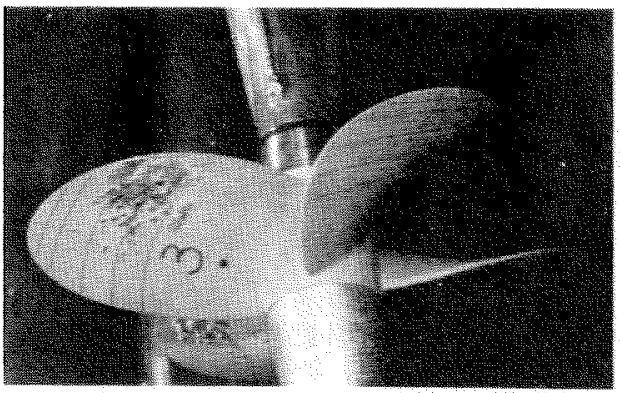

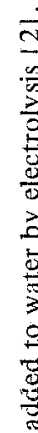
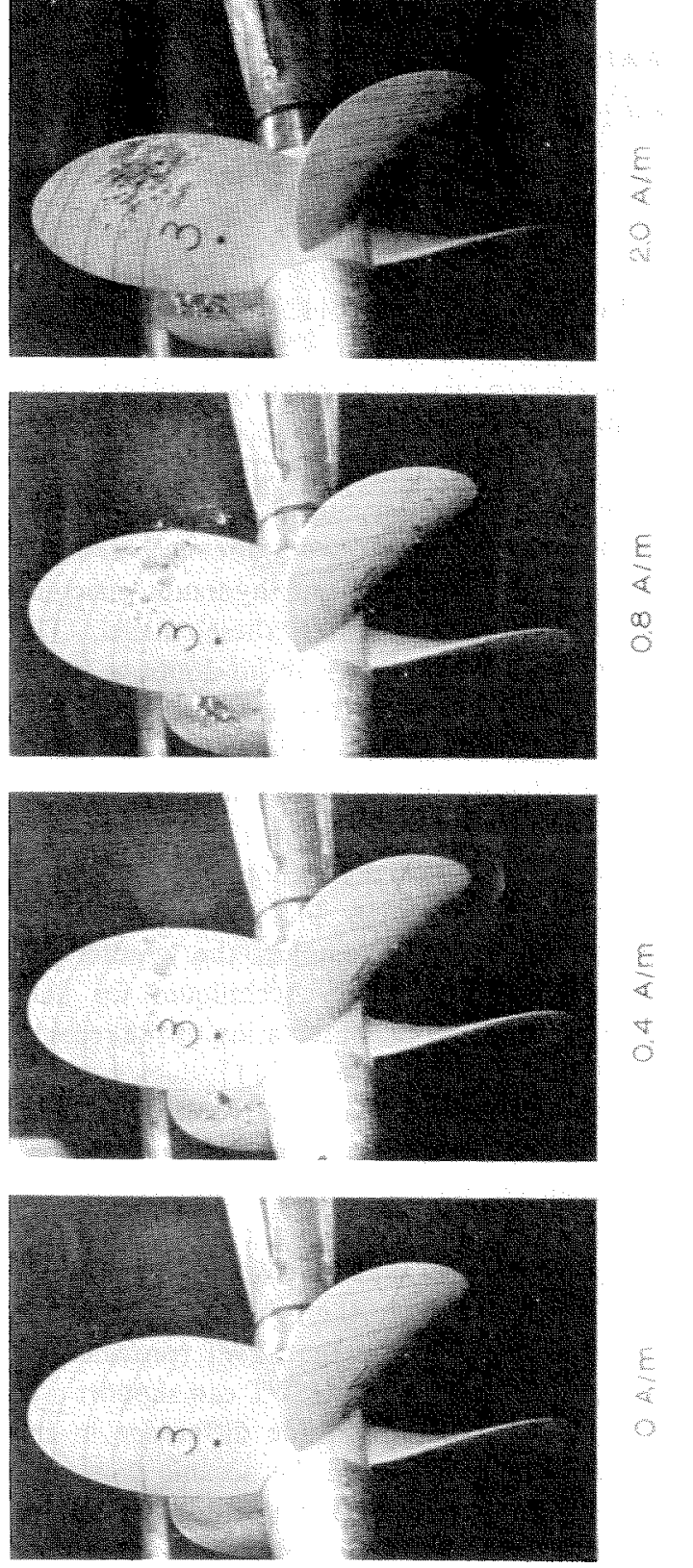


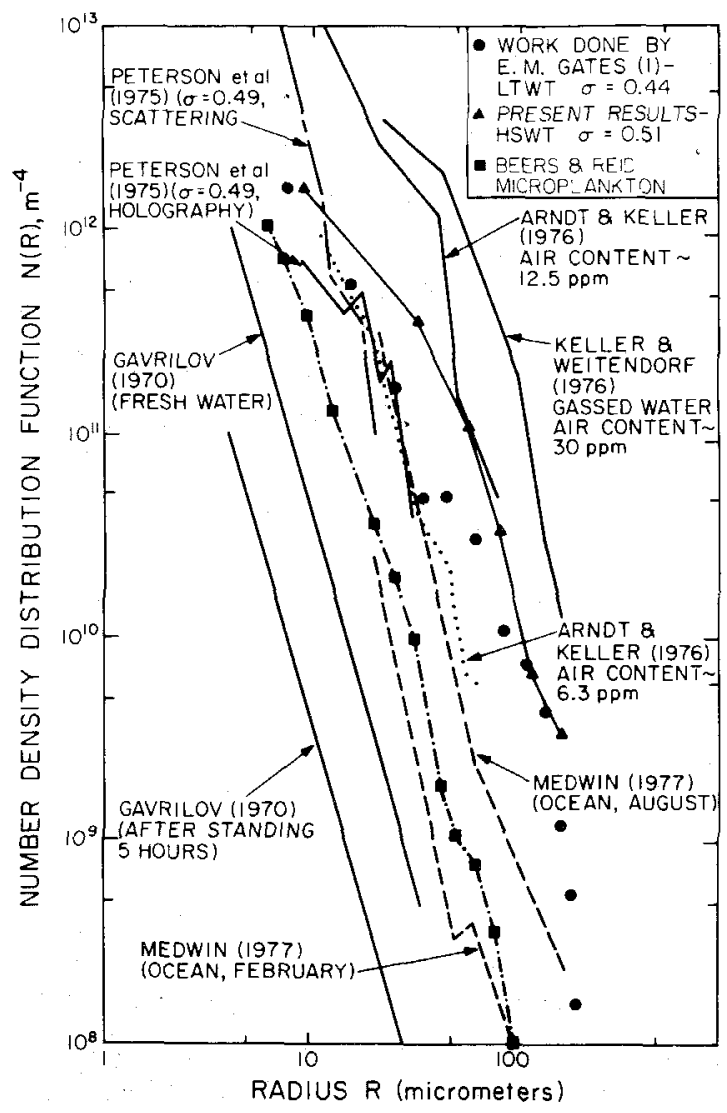

Figure 2. Nuclei distributions from various sources. The data of the HSWT and Beers et al. [5] are superposed on a graph presented in [6].

\section{Nuclei and cavitation inception}

Our own interest has been to identify the type, the location and the role of the nuclei in the cavitation process in steady flows past bodies. Following other investigators in this country and elsewhere (see Gates [6] for details) we have opted for the holographic system which provides one with the ability to record details to about 10 micrometers diameter. The details of the holocamera and the reconstruction system used herein are illustrated in Figures 3 and 4 respectively. This is an in-line system with a small modification in the reconstruction procedure that enables us to use it as a schlieren recording system as well [7].

Figure 5 is a photograph of cavitation inception on a hemispherical head form body with a downstream facing step $(1 \mathrm{~mm}$ height) at the tangency point. The flow in this region is visualized by heating the body about one 


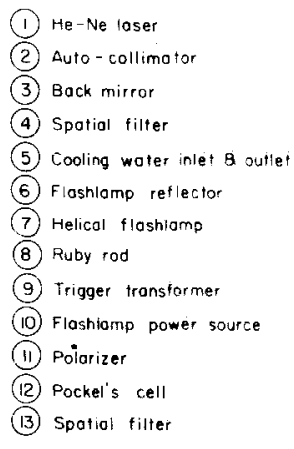

(9) Trigger tronstormer

(10) Flashiomp power source

(12) Pockel's cell

(13) Spatiol filter
(14) Front mirror
(15) Beam splitter
(16) Pin diode
(17) Oscilloscope
(18) Microscope objective
(19) Sparial filter (pin holel
(20) Collimating lens
(21) Pockel's cell power source
(22) Auto collimator light source
(23) Recording film
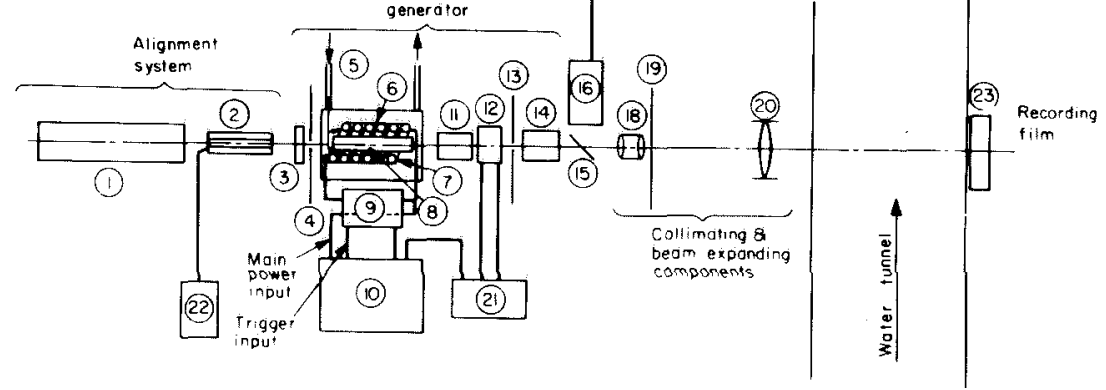

Figure 3. Diagram of the holocamera.

degree Centigrade and reconstructing the hologram of this flow as a schlieren picture (Figure 6) that shows clearly the laminar separation, the transition in the shear layer, and part of the turbulent mixing zone. Holograms that are recorded just prior to the formation of visible cavitation reveal the appearance of a large number of microbubbles at the unstable part of the shear layer upstream of the reattachment zone as is shown in Figure 7. A detailed map of the bubble population in different sections of the flow field including part of the background 'free stream', the separated zone and a small region upstream is demonstrated in Figure 8. This map provides us with the

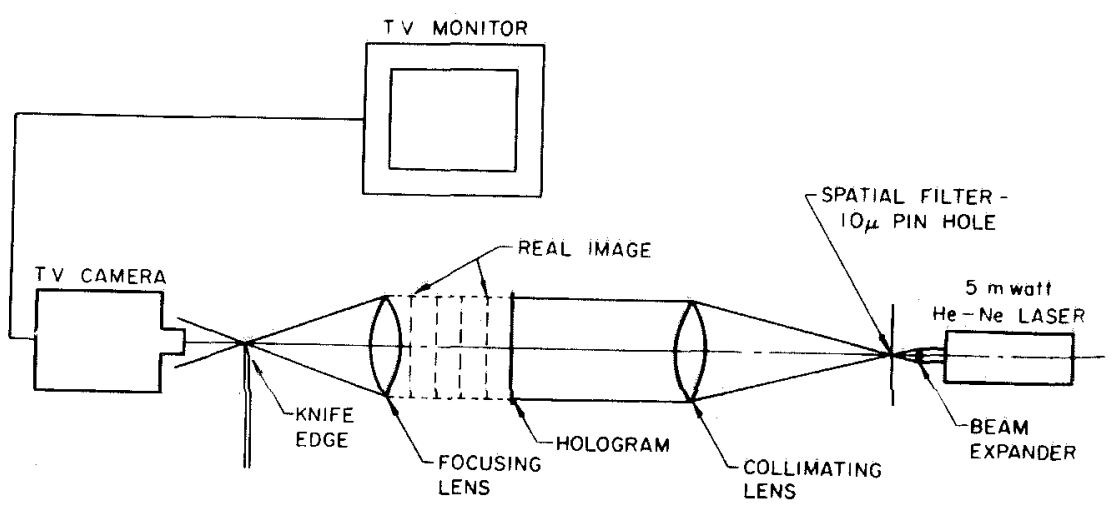

Figure 4. Diagram of the schlieren reconstruction system. 


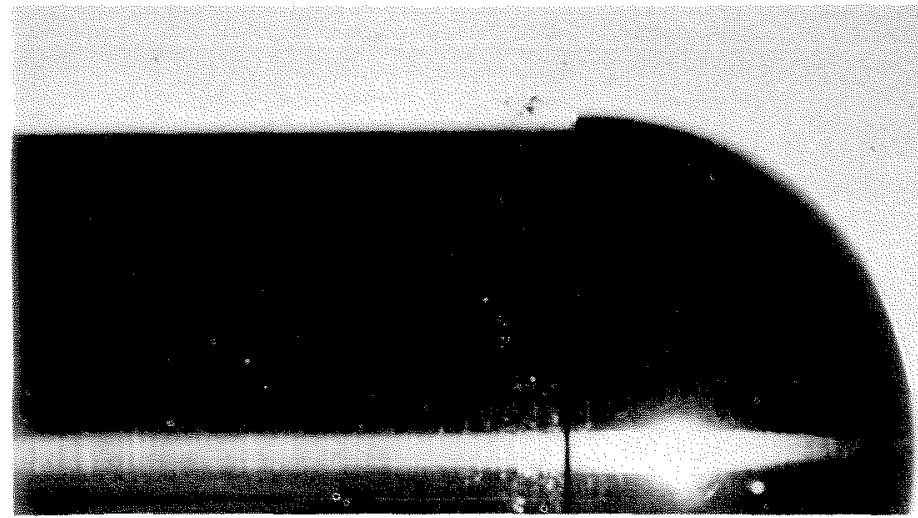

Figure 5. A photograph of cavitation inception on the hemispherical step body.

supply of free stream nuclei and the growing bubble population in the turbulent mixing zone during the transition from fully wetted flow to visible cavitation.

A schlieren-reconstructed hologram of the separated zone on the wellobserved hemispherical head form body is presented in Figure 9. A hologram of the flow field (Figure 10) reveals the formation of a cluster of microbubbles upstream of and at the reattachment zone just prior to inception of macroscopic cavitation. None of these bubbles are attached to the surface of the body; their sizes vary from 10 to 150 micrometers diameter.

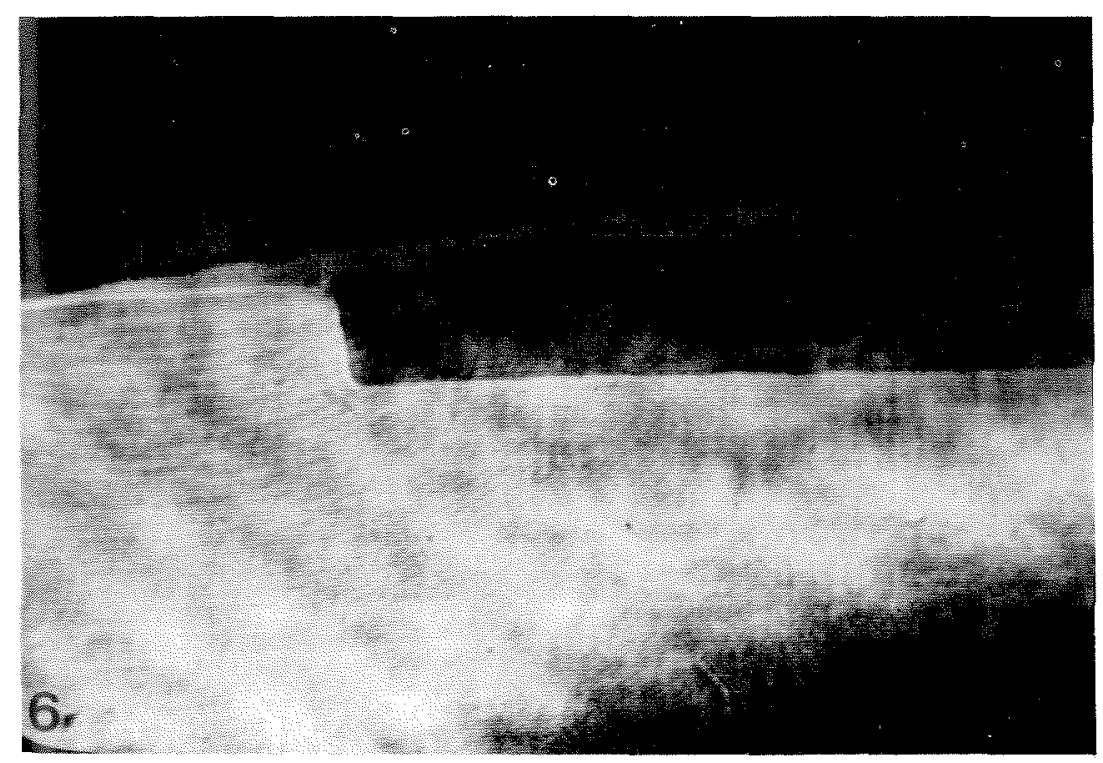

Figure 6. A schlieren reconstructed hologram on the surface of the hemispherical step body. $\operatorname{Re}_{D}=1.74 \times 10^{5}$. 


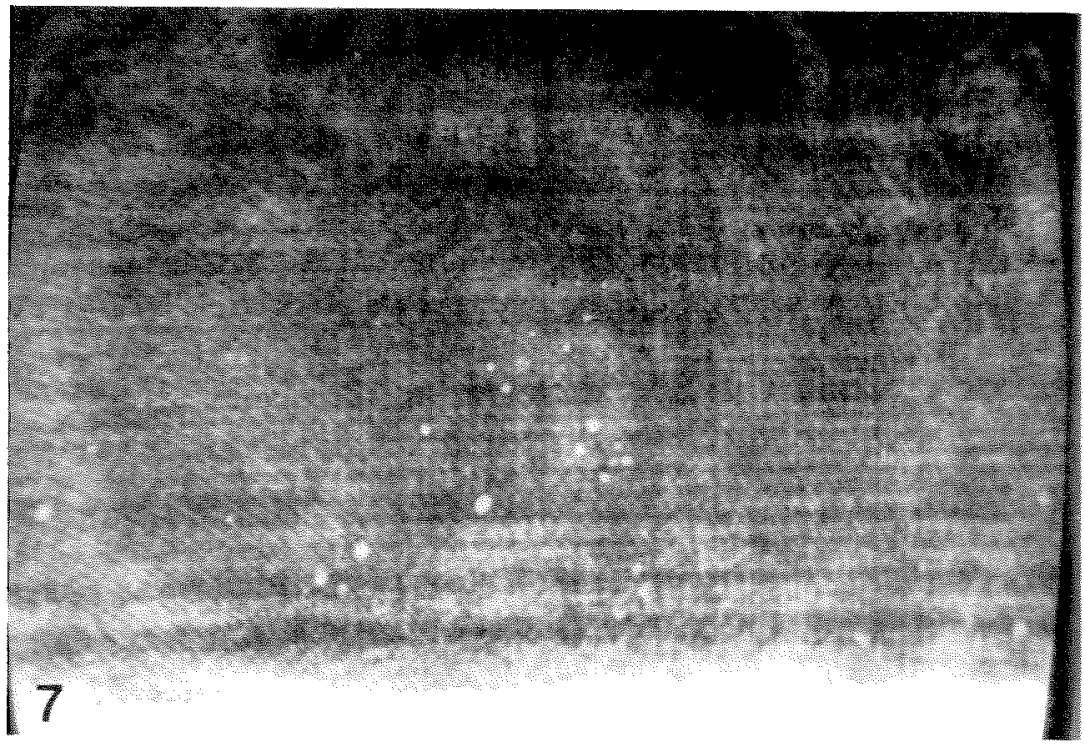

Figure 7. A hologram showing the appearance of microbubbles in the turbulent mixing zone extending from 3.5 to $10.5 \mathrm{~mm}$ behind the step just prior to the inception of visible cavitation. $\operatorname{Re}_{D}=2.78 \times 10^{5} ; \sigma=1.07$.

Bubble Diameter in microns

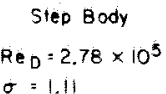

\begin{tabular}{|c|c|c|c|c|c|c|c|}
\hline & $0-20$ & $20-50$ & $50-75$ & $75-100$ & $100-150$ & $150-200$ & $200-250$ \\
\hline$a$ & 11 & 1 & 2 & - & - & & \\
\hline$b$ & 7 & 1 & 1 & & & & \\
\hline$c$ & 10 & - & 1 & & & & \\
\hline$d$ & 12 & 1 & 1 & 1 & & & \\
\hline$e$ & 10 & 2 & 1 & - & - & - & 1 \\
\hline 1 & 4 & 1 & - & - & - & 2 & \\
\hline$g$ & 8 & - & 1 & & & & \\
\hline
\end{tabular}

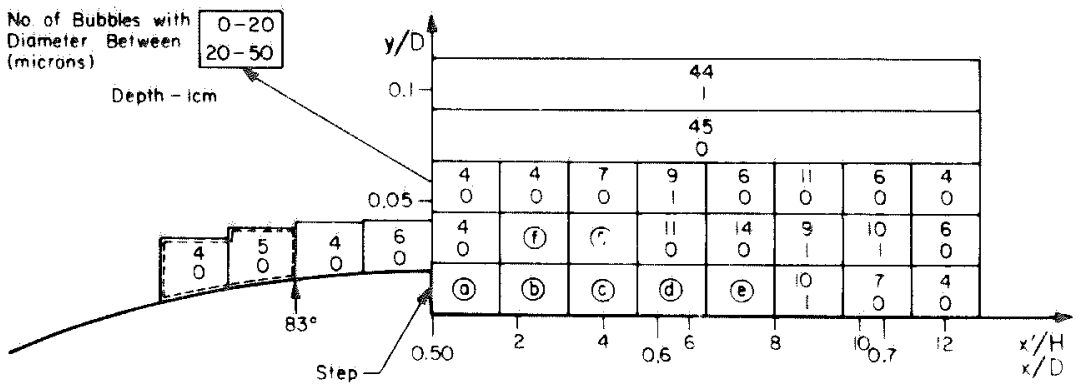

Figure 8. A map of the bubble population in the neighborhood of the step prior to the appearance of macroscopic cavitation. $\operatorname{Re}_{D}=2.78 \times 10^{5} ; \sigma=1.11\left(\sigma=\left(p_{\infty}-p_{0}\right)\right)$ $\frac{1}{2} \rho U^{2}, p_{\infty}$ being the ambient pressure in the fluid, $p_{v}$ the vapor pressure, $\rho$ the density and $U$ the flow speed). 


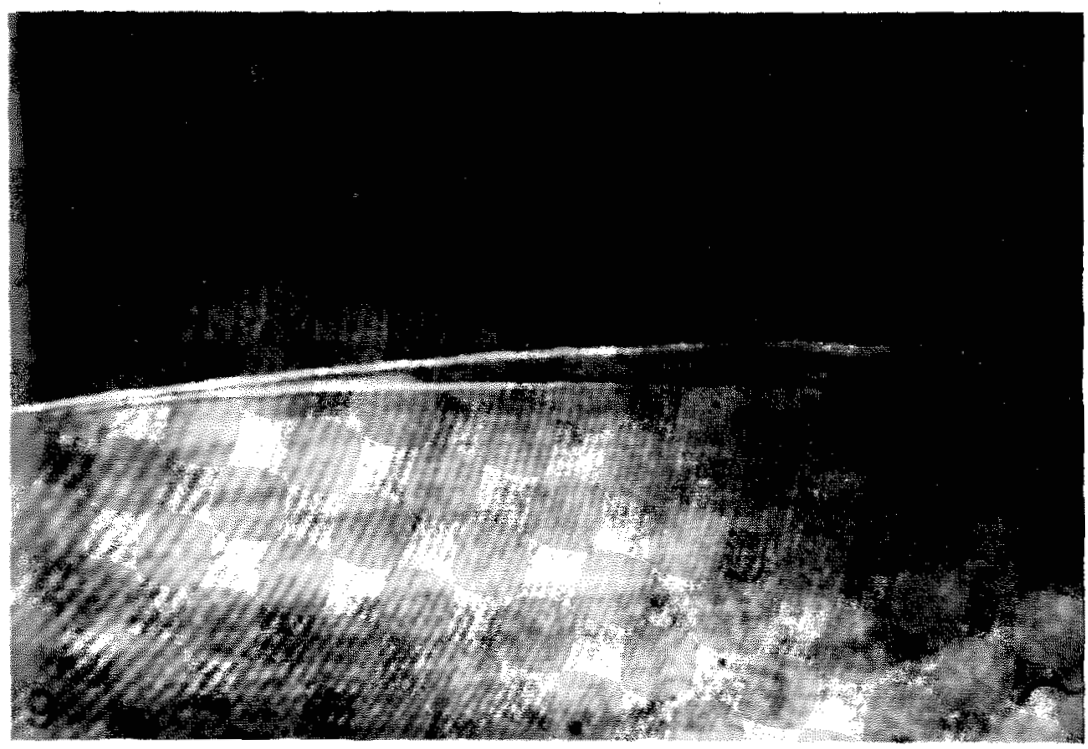

ligure 9. A schlieren-teconstructed hologram of the separation zone on the surface of the hemispherical body. $\operatorname{Re}_{D}=1.79 \times 10^{5}$.

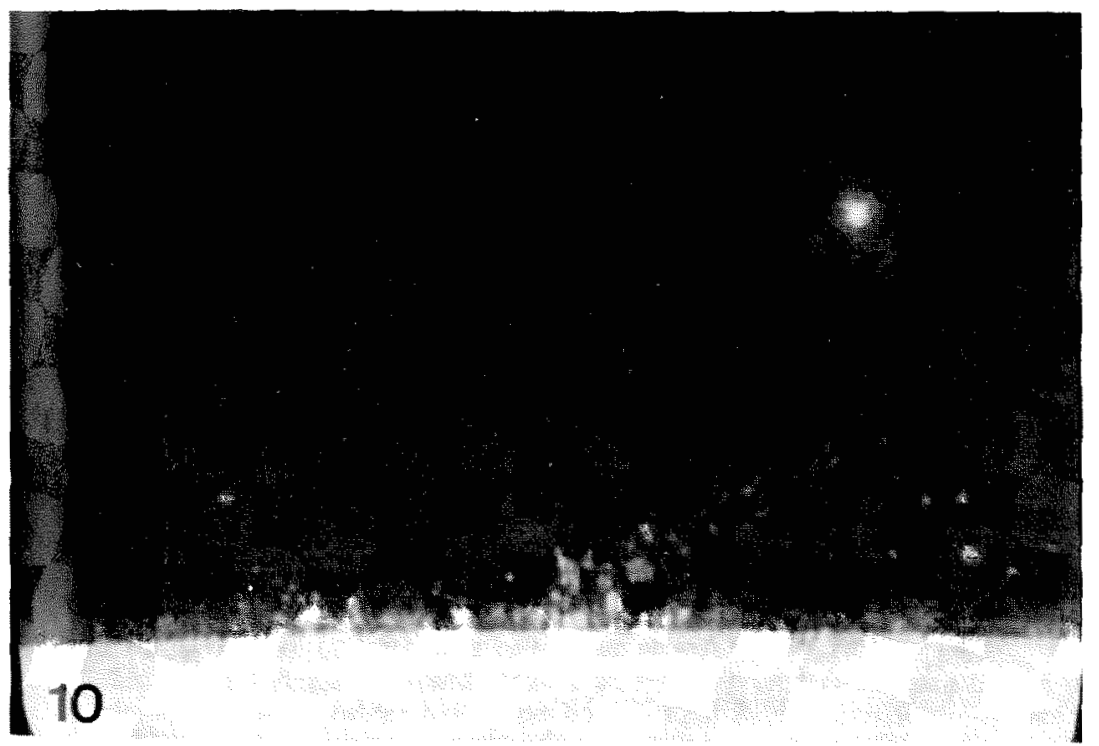

Figure 10. Inception of cavitation on the surface of the hemispherical body. $\operatorname{Re}_{D}=$ $3 \times 10^{5} ; \sigma=0.61$. The photograph covers the region between $x / D=0.593$ and 0.607 ( $x$ being the axial distance and $D$ is the body diameter). 
Similar phenomena can be observed in the flow around a flat blunt face body [7], characterized by a much larger separation region; that is a large number of microscopic bubbles appear in turbulent mixing zone just prior to the formation of macroscopic cavities.

\section{Nuclei control and future plans}

Being an important scaling factor makes the control of free stream nuclei population an essential part of any cavitation test. Experiments in the Caltech LTWT shown in Figure 11 reveal, on the one hand, almost an order of magnitude difference in the bubble population at the same cavitation index. Whereas, on the other hand, even at sharply different cavitation indices, 0.61 vs 1.7 the bubble density is almost equal (the air content is kept constant, $10 \mathrm{ppm}$ ). These somewhat 'surprising' phenomena are due to the procedures that preceded the recording of the hologram. In one case, that is represented by the lower $N(R)$ distribution in Figure 11 the tunnel was run at $\sigma=5.5$ for at least ten minutes before lowering the pressure whereas in the larger case the cavitation index was kept equal to 2.5. The final $\sigma$ to which the tunnel is brought seems to be much less

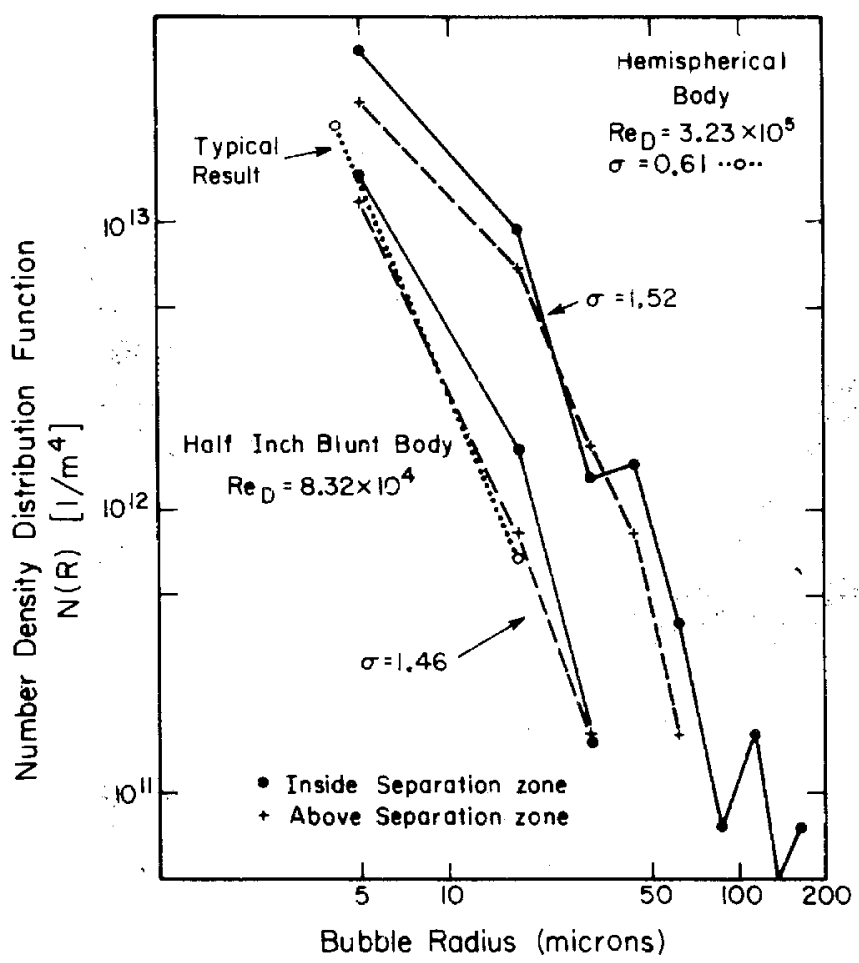

Figure 11. Bubble distribution in the Caltech LTWT determined by holography. 
important than the steady state conditions prior to pressure reduction. These results (and they are representative of detailed experiments that lead to the same conclusion [7]) suggest the proper procedure for a repeatable cavitation test when the nuclei population is an important parameter. Note that the lowest $N(R)$ found in the LTWT is still considerably larger than the oceanic values that are presented in Figure 1.

There are very few undisturbed measurements of $N(R)$ in natural waters or even in the circuits of large hydraulic structures. Due to the long-standing interest in our marine applications of cavitation we think that it is of some value to pursue the idea of increasing our overall knowledge of the natural science of particulates (debris, planktonic and microbubbles) in natural waters. To this end we have adapted the components shown in Figure 3 into an underwater holocamera. Our goal is to make an additional contribution to the available data of the nuclei distribution in the ocean that will be used in the future for modeling of the natural waters. We also plan to coordinate these observations while the simpler liquid quality meter designed in the Delft Hydraulic Laboratory [11]. These results will assist in judging whether different oceanic particulates are a good source of cavitation nuclei.

\section{Acknowledgment}

This work was supported by Naval Sea Systems Command General Hydromechanics Research Program administered by the David Taylor Naval Research and Development Center under Contract No. N00014-75-C-0378. We would also like to thank Barbara Katz (Sea Grant, Cal State University of Long Beach) for helpful information.

\section{References}

1. Acosta AJ and Parkin BR (1975) Cavitation inception - A selective review. J Ship Res 19 (4): 193-205.

2. Acosta AJ and Parkin AR (1980) Report of the ATTC Cavitation Inception Committee Proc 19th Am Towing Tank Conf 2: 829-858.

3. Arndt REA (1981) Cavitation in fluid machinery and hydraulic structures. Am Rev Fluid Mech 13: 273-328.

4. Billet ML and Gates EM (1979) A comparison of two optical techniques for measuring cavitation nuclei. International Symposium on Cavitation Inception, ASME. New York.

5. Beers JR, Reid FMH and Steward GL (1973) Microplankton of the North Pacific Central Gyre. Population Structure and Abundance, June 1973. Int Revue ges Hydrobiol 60 (5): 607-638.

6. Gates EM and Acosta AJ (1978) Some effects of several free-stream factors on cavitation inception of axisymmetric bodies. 12th Symp Naval Hydrodynamics. Washington DC: 86-108.

7. Katz J (1981) Some cavitation phenomena associated with separated flows. $\mathrm{Ph} D$ Thesis, California Institute of Technology, Pasadena, CA (in preparation).

8. Knapp RT, Daily JW and Hammitt FG (1970) Cavitation. New York: McGraw-Hill Book Company. 
9. Kuiper G (1981) Cavitation Inception on Ship Propeller Models. Ph D Thesis, Technische Hogeschool Delft. Wageningen: Veenman.

10. Medwin H (1977) In situ acoustic measurements of microbubbles at sea. J Geophys Res 82 (6): $921-976$.

11. Oldenziel DM (1979) Bubble cavitation in relation to liquid quality. Ph D Thesis Technical University Twente. Delft: Delft Hydraulic Laboratory, Publication No. 211.

12. Plesset MS (1969) The tensile strength of liquids. Cavitation State of Knowledge. ASME: $15-25$.

13. Stewart GL and Bears JR (1973) Proc Soc Photo-Opt Inst Engrs 41: 183-188. 